\title{
Numerical Study of Axisymmetric Flow and Heat Transfer in a Liquid Film over an Unsteady Radially Stretching Surface
}

\author{
Azeem Shahzad, ${ }^{1}$ Uzma Gulistan, ${ }^{1}$ Ramzan Ali ${ }^{2},{ }^{2}$ Azhar Iqbal, ${ }^{3}$ Ali Cemal Benim, ${ }^{4}$ \\ Muhammad Kamran, ${ }^{5}$ Salah Ud-Din Khan $\mathbb{D}^{6},{ }^{6}$ Shahab Ud-Din Khan, ${ }^{7}$ and Aamir Farooq ${ }^{8}$ \\ ${ }^{1}$ Department of Basic Sciences and Humanities, University of Engineering and Technology, Taxila 47050, Pakistan \\ ${ }^{2}$ Department of Mathematics and Natural Science, School of Arts and Sciences, University of Central Asia, 310 Lenin Street, \\ 722918 Naryn, Kyrgyzstan \\ ${ }^{3}$ Department of Mathematics, University of Wah, Islamabad, Pakistan \\ ${ }^{4}$ Center of Flow Simulation (CFS), Faculty of Mechanical and Process Engineering, Dusseldorf University of Applied Sciences, \\ Münsterstrasse 156, Rather Strasse 23 b, 40476 Dusseldorf, Germany \\ ${ }^{5}$ Department of Mathematics, COMSATS University Islamabad, Wah Campus, Rawalpindi 47040, Pakistan \\ ${ }^{6}$ Sustainable Energy Technologies (SET) Center, College of Engineering, King Saud University, P.O. Box 800, Riyadh 11421, \\ Saudi Arabia \\ ${ }^{7}$ National Tokamak Fusion Program, P.O. Nilore, Islamabad 45650, Pakistan \\ ${ }^{8}$ College of Mathematics and Statistics, Chongqing University, Chongqing 401331, China
}

Correspondence should be addressed to Ramzan Ali; ramzan.ali@ucentralasia.org

Received 7 January 2020; Revised 25 March 2020; Accepted 24 April 2020; Published 28 August 2020

Academic Editor: Babak Shotorban

Copyright (c) 2020 Azeem Shahzad et al. This is an open access article distributed under the Creative Commons Attribution License, which permits unrestricted use, distribution, and reproduction in any medium, provided the original work is properly cited.

\begin{abstract}
The main emphasis on this paper is to analyze the axisymmetric flow and heat transfer in a liquid film over an unsteady radially stretching surface in the presence of a transverse magnetic field. The similarity transformations are used to reduce the highly nonlinear governing partial differential equations for momentum and energy into a set of ordinary differential equations. A numerical scheme is developed for the reduced nonlinear differential equations for the velocity and temperature fields. The literature survey shows that the present problem of thin film flow over a radially stretching sheet has not been studied before. The features of the flow and heat transfer characteristic for different values of governing parameters such as unsteadiness parameter, Prandtl number, Eckert number, and magnetic parameter are thoroughly examined. This study noticed that, by increasing the magnetic parameter and unsteadiness parameter, film thickness decreases.
\end{abstract}

\section{Introduction}

The study of laminar boundary layer flow and heat transfer across a thin liquid film gains enormous interest among many researchers. This tremendous amount of attention further enhances to explore increasingly industrial application owing to stretching phenomena, to be specific, for example, the designing of various heat exchangers, coating processes, paper production, annealing and thinning of cooper wire, continuous stretching of plastic films and artificial fibers, metal, polymer extrusion, and metal spinning.
The boundary layer along material handling conveyors and the extrusion from the die is generally drawn and simultaneously stretched into sheet.

The problem of stretching sheet for different cases of fluid flow has been analyzed by different researchers. Sakiadis [1] in his seminal work initiated the study of boundary layer flow over a continuous solid surface by taking constant speed. Since then, many researchers contributed to the stretching sheet area due to its aforementioned industrial applications. Crane [2] modeled flow configuration and obtained an exact solution for an extension of a similar problem. He modeled and analyzed a steady 
two-dimensional boundary layer flow over stretching sheet, which moves in its own plane with a distance from the slit.

An enormous amount of work has been done in the direction of stretching sheet by number of researchers; just to mention a few, authors in [3-5] studied various aspects of stretching sheet such as the heat/mass and momentum transfer of a semi-infinite fluid layer driven by a continuous stretching sheet. They contributed to diverse range of geometries related to stretching sheet, for example, continuous stretching of sheet, radially stretching fixed sheet, and vertical stretching sheets with Newtonian and non-Newtonian behaviors of different kind. Furthermore, they discussed peculiar characteristics of heat and mass transfer with magnetohydrodynamic flow together with suction/injection phenomena. Shahzad and Ali [6, 7] in a series of articles proposed approximate analytic solutions with a thorough investigation of non-Newtonian fluid model of power law over stretching sheets. In addition, they discussed the flow in heat transfer together with the convective boundary condition over a vertical wall. Andersson et al. [8] worked on unsteady heat transfer problems over a time-dependent stretching sheet. They introduced new similarity solution for the temperature field. Although finding an analytic solution for PDEs model is always hard due to difficulty of the model and nonlinear terms in the equations, in this scenario, one of those rare studies includes an exact analytical solution by Wang [9]. He found an exact solution for two-dimensional flow past a stretching sheet. He compared his obtained solution of a liquid film over an unsteady stretching surface with HAM solutions; both show a great deal of agreement. Abel et al. [10] studied heat transfer rate in a liquid film over an unsteady stretching surface including viscous dissipation in the presence of an external magnetic field with specific surface temperature. Aziz and Hashim [11, 12] in a couple of articles studied the influence of internal heat generation on flow and heat transfer in a thin liquid film on an unsteady stretching sheet; they assumed and modeled a general surface temperature for this purpose. Nandeppanavar et al. [13] considered the combined effects of viscous dissipation, thermal radiation, and nonuniform heat source/sink on the presence of magnetic field. Salleh et al. [14] considered the steady boundary layer flow and heat transfer over a stretching sheet with Newtonian heating problem of MHD flow. It is an important field for exploration due to abundant practical applications. The actual interest in MHD flow began around 1918, when Hartmann [15] invented the electromagnetic pump. Meanwhile, the viscous MHD flow over an infinite flat plate has received great attention. The mathematical analysis and development of new schemes and algorithms grow quite significantly. Ishak et al. [16] studied laminar MHD flow and heat transfer due to continuously stretching plate immersed in an electrically conducting fluid. Abel et al. [17] studied the MHD boundary layer flow and heat transfer characteristic of a laminar liquid film over a flat impermeable stretching sheet in the presence of a nonuniform heat source/sink. Abbas et al. [18] analyzed the unsteady MHD boundary layer flow and heat transfer of an incompressible rotating viscous fluid over a stretching continuous sheet. Hayat et al. [19] considered the MHD flow and heat transfer characteristic for the boundary layer flow over a permeable stretching sheet with velocity and thermal slip conditions.
Chen et al. [20] studied the MHD flow and heat transfer of an electrically conducting viscoelastic fluid past a stretching surface and discussed the effects of joule and viscous dissipation. Fadzilah et al. [21] studied the MHD boundary layer flow and heat transfer of a viscous and electrically conducting fluid over a stretching sheet with an induced magnetic field. In the process of understanding of MHD flow, Labropulu [22] discussed the effect of transverse magnetic field on an infinite plate and studied the unsteady stagnation point flow of a Newtonian fluid. Further analysis of the flow over a stretching sheet in the presence of transverse magnetic field with heat source/sink has been performed in [23]. This study deals with the numerical study of unsteady laminar MHDs boundary layer flow and heat transfer of incompressible, viscous, and electrically conducting fluid. Bhukta et al. [24] investigated the dissipation effects on MHD-mixed convection unsteady flow of an electrically conducting fluid over a stretching sheet embedded in a porous medium subject to transverse magnetic field. Gnaneswara Reddyet al. [25] presented the unsteady MHD boundary layer flow and heat transfer of a fluid over a stretching sheet in the presence of viscous dissipation and heat source. A similar model for micropolar fluid flow is studied in Anantha Kumar et al. [26-29]. They provided simultaneous solution for slip flow across convective surfaces in MHD flow model with varying heat flux [26], thermal radiation [27], and irregular heat source and sink [28] with thin film flow and viscous dissipation [29] with modified heat flux. In a series of articles [30-34], Anantha Kumar et al. [30-34] extensively studied a number of physical aspects on steady/unsteady MHD flow in heat transfer. Their investigation includes a diverse range of geometrical models, such as stretching, nonstretching, coagulated sheet, porous media, flow past cone, and inclined domain. In this intensive literature survey, to the best of our knowledge, we could not find a single attempt on thin film flow over a radially stretching sheet. The application of thin film flow in industrial applications motivated the authors to examine and develop a numerical solution for axisymmetric flow and heat transfer in Newtonian fluid. The authors studied the effects of magnetic field and the mechanism of heat transfer over a radially stretching sheet. The authors proposed a timedependent PDE model for momentum and energy equations. The system of PDE is transformed into system of ordinary differential equations using a chosen set of similarity transformations, which identically satisfy the conservation of mass. Then, these ODEs are solved numerically by using BVP4C in MATLAB. The effects of physical flow parameters such as magnetic parameter, unsteadiness parameters, Eckert number on skin friction coefficient, and Nusselt number are examined. The results of these studies are of great importance in viewpoint of desired properties of the outcome.

With this brief introductory section, the paper is outlined as follows: Section 1 will discuss a thorough mathematical formulation including the transformation of PDEs into ODEs. In addition, a schematic of flow and nomenclature of the diverse mathematical symbols are tabulated. In Section 2, the numerical scheme is developed for the ODEs. Section 3 summarized the results and discussion of potential pertaining parameters to analyze flow behavior, and finally, Section 4 shows a few concluding remarks. 


\section{Problem Formulation}

Let us consider two-dimensional unsteady MHD boundary layer flow of Newtonian fluid in a thin liquid film over a radially stretching sheet whose center is fixed. The sheet is coinciding with the plane at $z=0$ and is stretched along the radial direction with the velocity $U(r, t)=(b r /(1-\alpha t))$, where $b$ and $\alpha$ are both positive constants with dimension per time. $T \mathrm{~s}$ is the surface temperature of the stretching sheet and is defined as $T_{s(r, t)}=T_{0}-T_{\text {ref }}\left[b r^{2} / 2 v\right](1-\alpha t)^{-(3 / 2)}$, where $T_{0}$ is the slit temperature and $T_{\text {ref }}$ can be taken as a constant reference temperature such that $0 \leq T_{\text {ref }} \leq T_{0} . h$ is the thickness of the fluid film. Furthermore, the variable magnetic field of intensity $B(t)$ chosen in its special form as $B(t)=$ $B_{o}(1-\alpha t)^{(-1 / 2)}$ is applied along normal to the sheet. More details could be found on each parameters in Table 1.

The graphical representation of the considered physical model is given in Figure 1. The cylindrical polar coordinate system $(r, \theta, z)$ is taken for the mathematical modeling. Due to the rotational symmetry of the flow, all the physical quantities are independent of $\theta$ and the velocity field takes the form $v=[u(r, z), 0, w(r, z)]$, where $u$ and $w$ are the velocity components along the radial $r$ and axial $z$ direction, respectively.

Under the abovementioned assumptions, the basic governing equations for mass, momentum, and energy may be written as

$$
\begin{gathered}
\frac{\partial u}{\partial r}+\frac{u}{r}+\frac{\partial w}{\partial z}=0 \\
\frac{\partial u}{\partial t}+u \frac{\partial u}{\partial r}+w \frac{\partial u}{\partial z}=v \frac{\partial^{2} u}{\partial z^{2}}-\frac{\sigma B(t)^{2}}{\rho} u \\
\frac{\partial T}{\partial t}+u \frac{\partial T}{\partial r}+w \frac{\partial T}{\partial z}=\frac{\kappa}{\rho c_{p}} \frac{\partial^{2} T}{\partial z^{2}}+\frac{v}{c_{p}}\left(\frac{\partial u}{\partial z}\right)^{2}
\end{gathered}
$$

where $T$ represents the temperature of the fluid, $v$ indicates the kinematic viscosity, $\sigma$ indicates the electrical conductivity, $\rho$ indicates the density of fluid, $\alpha=\left(\kappa / \rho c_{p}\right)$ indicates the thermal diffusivity of the fluid, $B$ is variable magnetic field, $c_{p}$ indicates the specific heat at constant pressure, and $\kappa$ indicates the thermal diffusivity.

The associated boundary conditions are given by

$$
\begin{gathered}
u=U, \\
w=0, \\
T=T_{S}, \\
z=0 . \\
\frac{\partial u}{\partial z}=\frac{\partial T}{\partial z}=0, \\
w=\frac{d h}{d t}, \\
z=h .
\end{gathered}
$$

TABle 1: Nomenclature.

\begin{tabular}{lc}
\hline Symbol & Definition \\
\hline$(r, \theta, z)$ & Polar coordinates \\
$\alpha=\left(\kappa / \rho c_{p}\right)$ & Thermal diffusivity \\
$(u, v, w)$ & Velocity components \\
$B(t)$ & Applied magnetic field \\
$T$ & Temperature of the fluid \\
$T_{\text {ref }}$ & Reference temperature \\
$c_{p}$ & Specific heat at constant \\
$p$ & Pressure \\
$\psi(r, z)$ & Stokes stream function \\
$v$ & Kinematic viscosity \\
$\sigma$ & Electrical conductivity \\
$\rho$ & Density of fluid \\
$t$ & Temporal variable \\
$\operatorname{Re}$ & Local Reynolds number \\
$C_{f}$ & Local skin friction coefficient \\
$N u_{r}$ & Nusselt number \\
$\operatorname{Pr}$ & Prandtl number \\
$E c$ & Eckert number \\
$l$ & Differentiation w.r.t. $\eta$ \\
$\beta$ & Thickness of the film \\
$\theta$ & Dimensionless temperature \\
\hline
\end{tabular}

Proceeding with the analysis, we introduced the following similarity transformations:

$$
\begin{aligned}
\psi & =-r^{2} U \operatorname{Re}^{(-1 / 2)} f(\eta), \\
\theta(\eta) & =\frac{T_{\circ}-T(r, z)}{T_{\text {ref }}\left(b r^{2} /\left(2 v(1-\alpha t)^{(3 / 2)}\right)\right)}, \\
\eta & =\frac{z}{r} \operatorname{Re}^{(1 / 2)},
\end{aligned}
$$

where $\eta$ is the independent variable, $\psi(r, z)$ is the Stokes stream function such that $u=-((1 / r)(\partial \psi / \partial z))$ and $w=((1 / r)(\partial \psi / \partial r))$, and $\operatorname{Re}=(r U / v)$ is the local Reynolds number. The velocity components are obtained as

$$
\begin{aligned}
& u=U f^{\prime}(\eta), \\
& w=-2 U \operatorname{Re}^{(-1 / 2)} f(\eta) .
\end{aligned}
$$

The above-defined set of similarity transformations identically satisfied the law of conservation mass in equation (1) and the mathematical problem defined in equations (2)-(5) transforms into a set of ordinary differential equations together with the boundary conditions as

$$
\begin{aligned}
f^{\prime \prime \prime}-S\left(f^{\prime}+\frac{\eta}{2} f^{\prime \prime}\right)-f^{\prime 2}+2 f f^{\prime \prime}-M f^{\prime}=0 \\
\theta^{\prime \prime}(\eta)-\operatorname{Pr}\left(\frac{S}{2}\left(3 \theta+\eta \theta^{\prime}\right)+2 \theta f^{\prime}-2 f \theta^{\prime}\right)-2 \operatorname{Pr} E c f^{\prime \prime 2}=0 \\
f^{\prime}(0)=1 \\
f(0)=0 \\
\theta(0)=1 .
\end{aligned}
$$




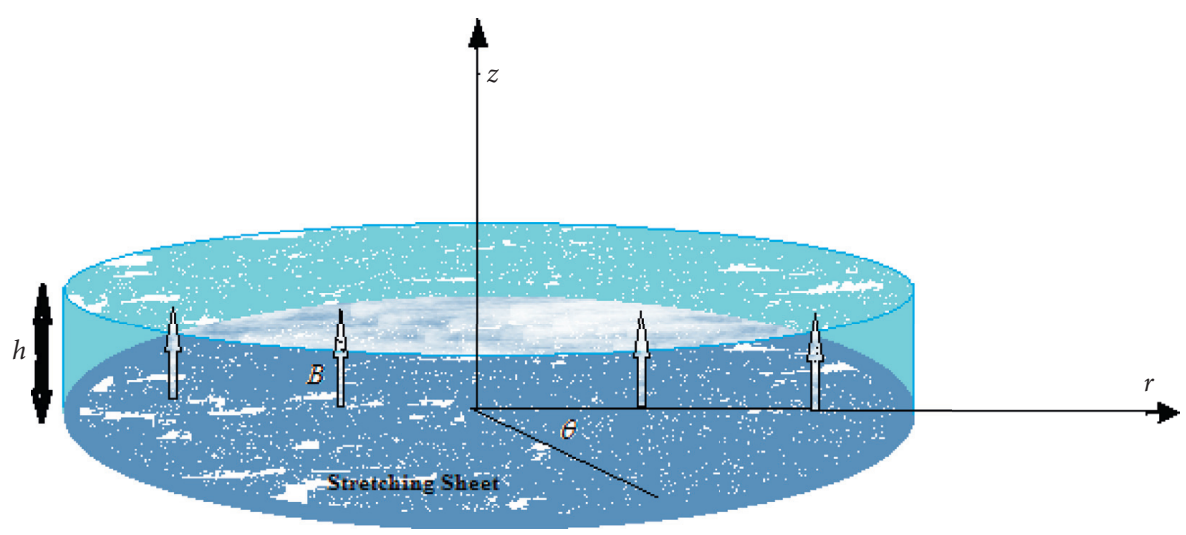

Figure 1: Schematic diagram of the flow over stretching sheet.

$$
\begin{aligned}
f^{\prime \prime}(\beta) & =0, \\
\theta^{\prime}(\beta) & =0, \\
f(\beta) & =\frac{S \beta}{2} .
\end{aligned}
$$

Here, $S=(\alpha / b)$ is the dimensionless measure of the unsteadiness parameter, $M=\left(\sigma B_{o}^{2} / \rho b\right)$ is the magnetic parameter, $\operatorname{Pr}=(\nu / \alpha)$ is the Prandtl number, $E c=$ $\left(U^{2} / C_{p} \nabla T\right)$ is Eckert number, and prime shows differentiation with respect to $\eta$. $\beta$ (unknown constant) is the dimensionless thickness of the film and is given as $\beta=(b / v(1-\alpha t))^{(1 / 2)} h$.

The rate at which film thickness varies can be obtained by differentiating $h$ with respect to $t$, in the form $(d h / d t)=(-\alpha \beta / 2)(v / b(1-\alpha t))^{(1 / 2)}$.

The important physical quantities of interest in this problem are the local skin friction coefficient and Nusselt number that are $C_{f}=\left(\mu(\partial u / \partial z)_{z=0} /\left(\rho U^{2} / 2\right)\right)$ and $\mathrm{Nu}_{r}=\left(-r / T_{\text {ref }}\right)(\partial T / \partial z)_{z=0}$, respectively.

In nondimensional form, these quantities can be written as

$$
\begin{array}{r}
\frac{1}{2} \operatorname{Re}^{(1 / 2)} C_{f}=f^{\prime \prime}(0), \\
2(1-\alpha t)^{(1 / 2)} \operatorname{Re}^{(-3 / 2)} \mathrm{Nu}_{r}=-\theta^{\prime}(0) .
\end{array}
$$

\section{Numerical Solution}

The nonlinear system of equations (8) and (9) is solved numerically after converting into initial value problem along with the boundary conditions (10) and (11) by employing the BVP4C package from MATLAB. In this method, the thirdorder nonlinear differential equation (8) and second-order differential equation (9) are reduced into a first-order differential equation as follows:

$$
\begin{aligned}
f & =y_{(1)}, \\
y_{(1)}^{\prime} & =y_{(2)}, \\
y_{(2)}^{\prime} & =y_{(3)},
\end{aligned}
$$

$$
\begin{aligned}
\theta & =y_{(4)}, \\
y_{(4)}^{\prime} & =y_{(5)}, \\
y_{(3)}^{\prime} & =\mathrm{My}_{(2)}+\mathrm{sy}(2)+\frac{S \eta}{2} y_{(3)}+\left(y_{(2)}\right)^{2}-y_{(1)} y_{(3)}, \\
y_{(5)}^{\prime} & =\operatorname{Pr}\left(\frac{S}{2}\left(3 y_{(4)}+\eta y_{(5)}\right)-2 y_{(1)} y_{(5)}+2 y_{(2)} y_{(4)}+2 E_{c}\left(y_{(3)}\right)^{2}\right) .
\end{aligned}
$$

The corresponding boundary conditions are

$$
\begin{aligned}
& y_{(1)}(0)=0, \\
& y_{(2)}(0)=y_{(4)}(0)=1, \\
& y_{(3)}(\beta)=y_{(5)}(\beta)=0, \\
& y_{(1)}(\beta)=\frac{S \beta}{2} .
\end{aligned}
$$

As equation (8) is the third-order differential equation with four boundary conditions, the initial guess for the value of $\beta$ is given by the program BVP4C in MATLAB and then the value of $\beta$ is adjusted, so that the condition $f(\beta)=$ $(S \beta / 2)$ holds. This is done on a hit and trial basis.

\section{Results and Discussion}

This study provides a new paradigm in the area of thin film. The objective of the upcoming discussion is to effectively develop a clear understanding of the governing parameters such as unsteadiness $s$, magnetic parameter $M$, Eckert number Ec, and Prandtl number Pr. The numerical simulation together with an analytical computation for the nonlinear differential equations is developed. Furthermore, we considered the analysis of dimensionless parameters on unsteady MHD boundary layer flow with heat transfer over a stretching surface. The effects of magnetic parameter $M$ on velocity profile are shown in Figure 2 . We observed that the Lorentz force acts as a retarding force. In consequence, the magnetic parameters reduced the velocity profile. In contrast, these retarding forces enhance the frictional resistance, which end up opposing the fluid motion. In principle, the velocity boundary layer thickness gets reduced and the 


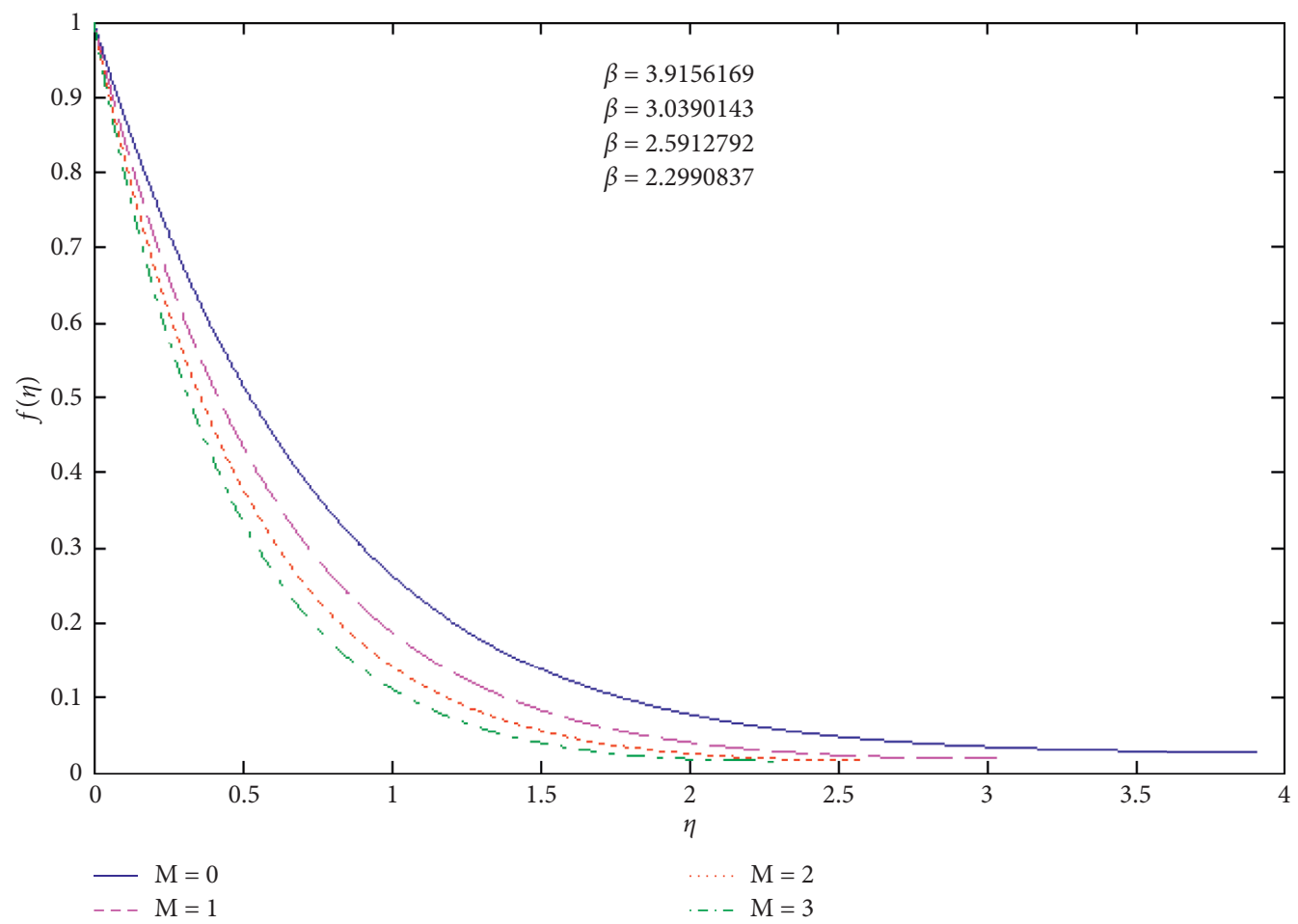

Figure 2: Effect of magnetic parameter on velocity profile.

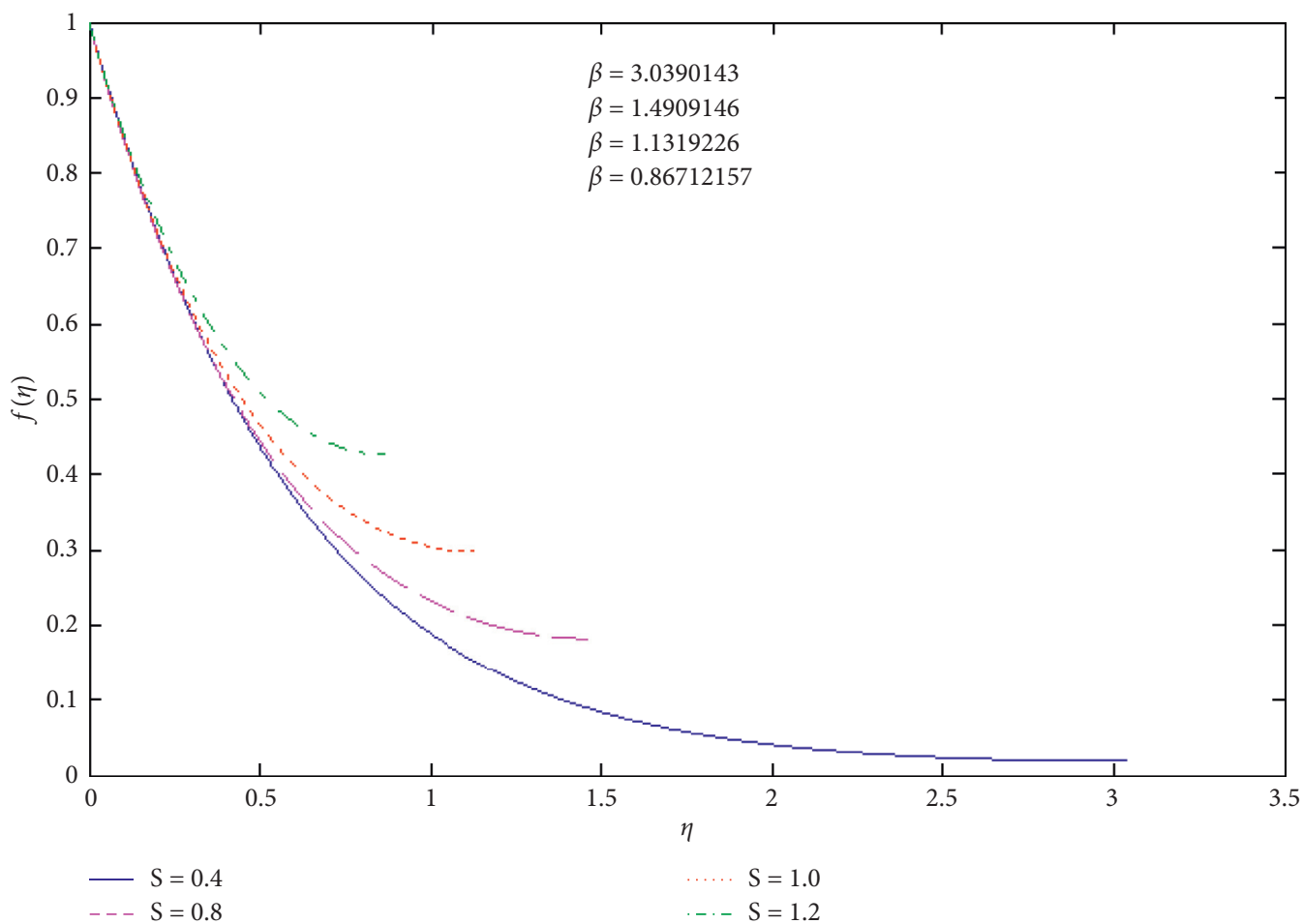

Figure 3: Effect of unsteadiness parameter on velocity profile.

temperature distribution increases with an increase in magnetic parameter. Figure 3 illustrates the effect of unsteadiness parameter $S$ on the velocity profile that leads to a decrease of the velocity with the increasing value of unsteadiness parameter. This shows that the boundary layer thickness becomes thicker for the larger amplitude of unsteadiness parameter. Figure 4 depicts the temperature distribution for different values of Eckert number (Ec). This 


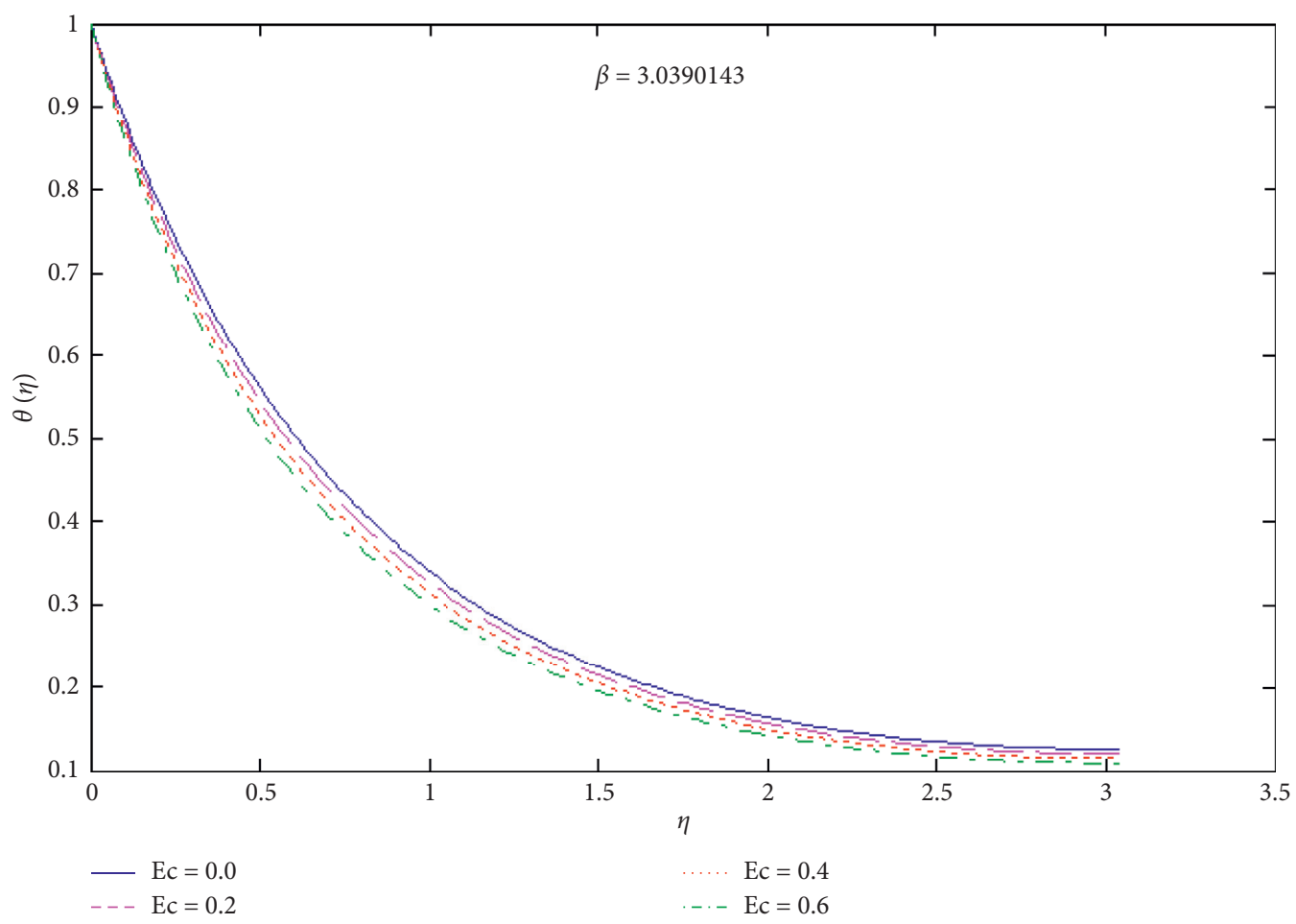

FIgURE 4: Variation of Eckert number on temperature profile.

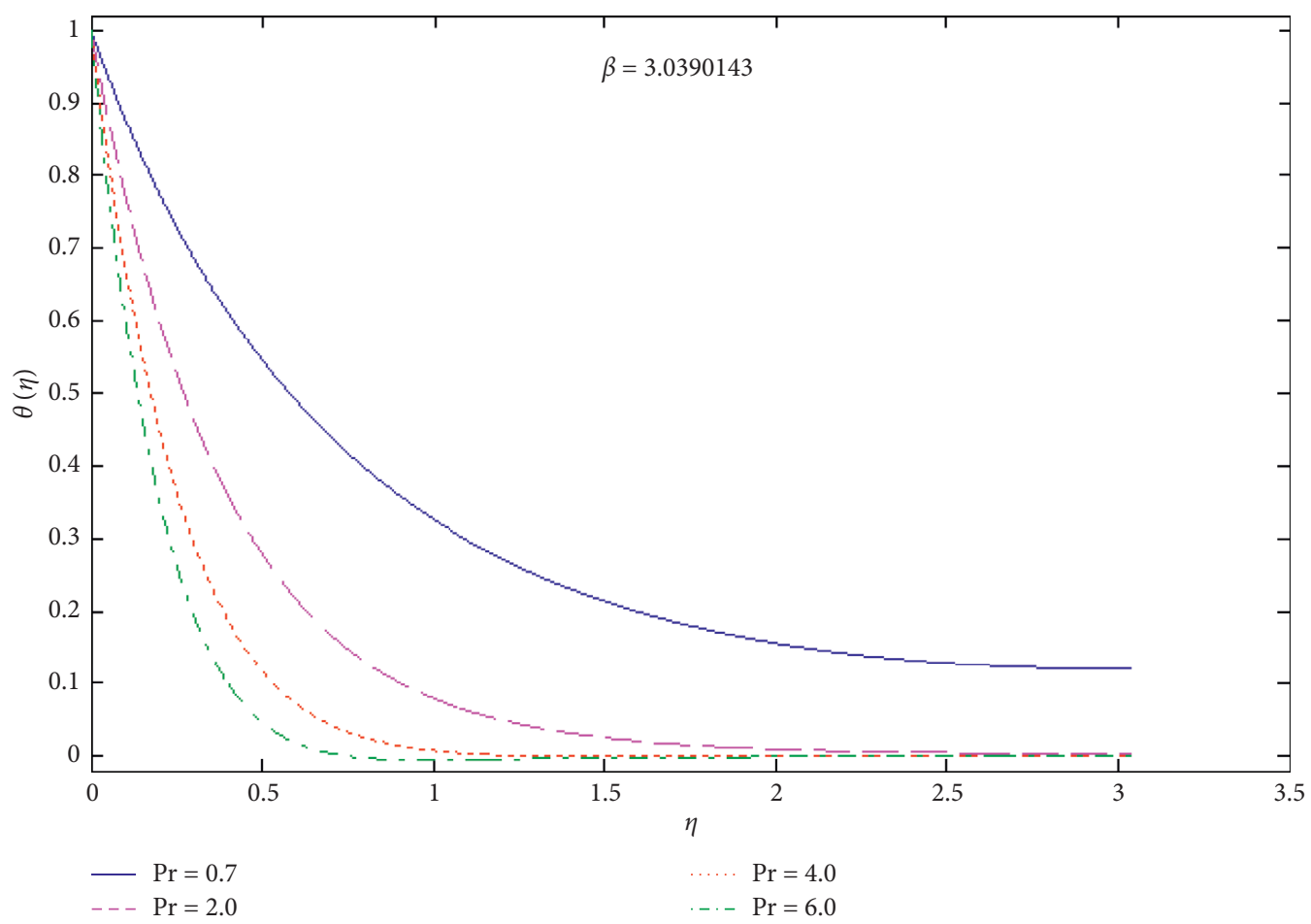

Figure 5: Effect of Prandtl number on temperature distribution.

physical parameter expresses the relationship between the kinetic energy and internal energy. Figure 5 shows the results of Prandtl number over temperature distribution. One can observe that an increase in dissipation reduced the thermal boundary layer. Prandtl number defines the ratio of momentum diffusivity to thermal diffusivity. In case of smaller values of Prandtl number, the boundary layer thickness increases, further causing a reduction in heat transfer. Figure 6 


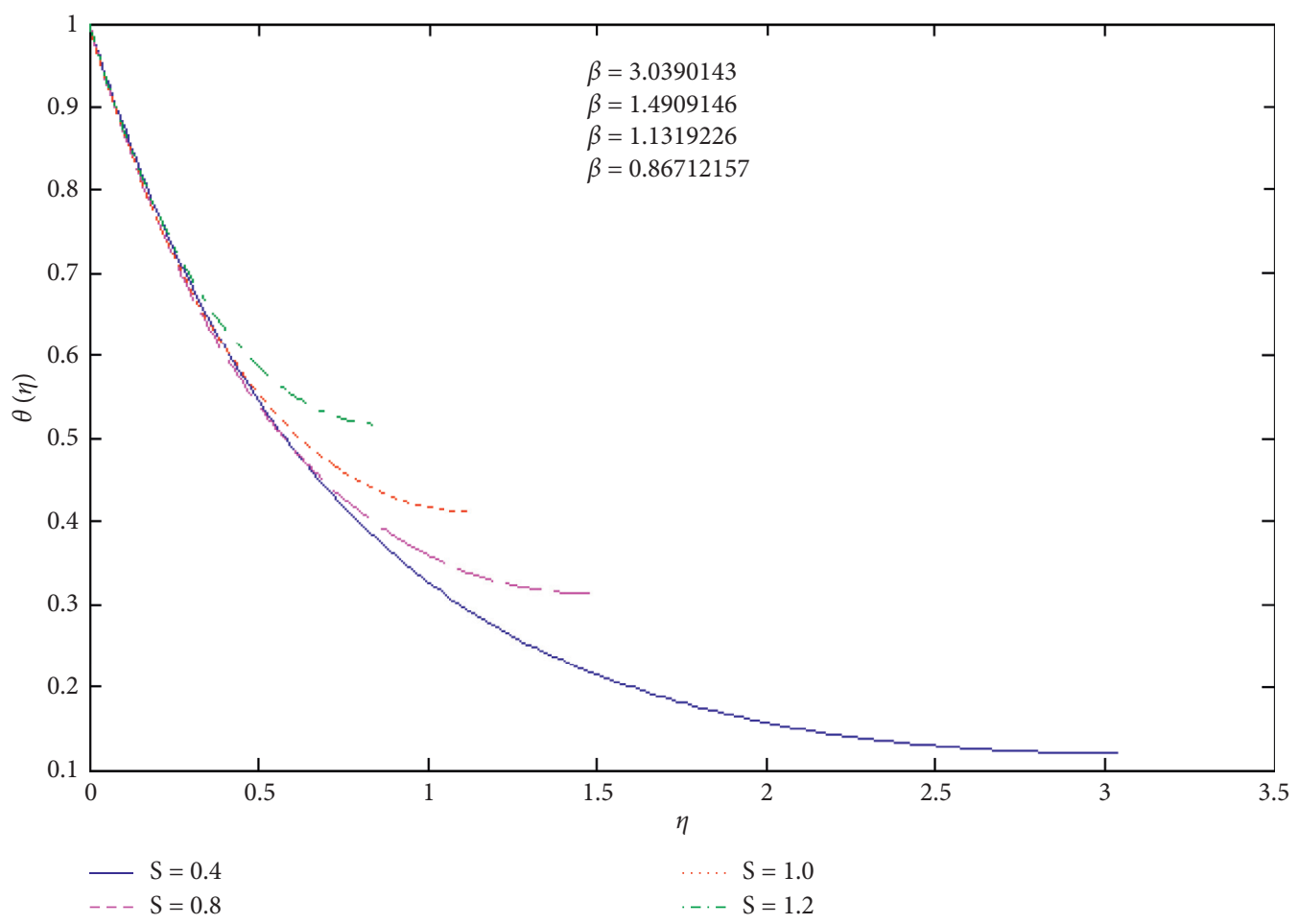

FIGURE 6: Variation of unsteadiness parameter on temperature distribution.

TABLE 2: Effects of pertinent parameters on skin friction coefficient and heat transfer rate.

\begin{tabular}{lccccc}
\hline $\mathrm{M}$ & $\mathrm{S}$ & $\mathrm{Pr}$ & $\mathrm{Ec}$ & $f^{\prime \prime}(0)$ & $\theta^{\prime}(0)$ \\
\hline 0 & 0.4 & 0.7 & 0.2 & -1.2784767 & \\
1 & & & & -1.6237276 & \\
2 & & & & -1.9067150 & \\
1 & 0.8 & 0.7 & 0.2 & -1.674472 & -1.4105271 \\
& 1.0 & & & -1.6650959 & -1.4200617 \\
& 1.2 & & & -1.6186823 & -1.3936895 \\
1 & 0.4 & 0.7 & 0.2 & & -1.3214547 \\
& & 4 & & & -3.8102363 \\
& & 6 & & & -1.2318785 \\
1 & 0.4 & 0.7 & 0.0 & & -1.3214547 \\
1 & & & 0.2 & & -1.414869 \\
1 & & & 0.4 & & \\
\hline
\end{tabular}

depicts the effects of unsteadiness parameter on temperature distribution in which temperature decreases as the parameter $\mathrm{S}$ increases. It is evident that the unsteadiness parameter causes a substantial amount of increase in heat transfer.

Table 2 is formulated in order to observe the effects of different pertinent parameters on skin friction coefficient and dimensionless heat transfer rate at the surface. An increase in magnetic parameter $M$ and the unsteadiness parameter $\mathrm{S}$ causes an increase in the skin friction coefficient. In addition, the Prandtl number and the unsteadiness parameter influence increase in the heat transfer rate at the surface.

\section{Conclusion}

The problem of unsteady MHD boundary layer flow and heat transfer in a liquid film over a radially stretching surface is investigated numerically. The effects of the various governing parameters on velocity and temperature profile are examined. We conclude with the following remarks:

(i) An increment in values of unsteadiness parameter and magnetic parameter results in a decay of film boundary layer thickness

(ii) Velocity increases with an increase in unsteadiness parameter and decreases by enhancing the magnetic parameter

(iii) An increase in unsteadiness parameter results in quite substantial enhancement in thermal conductivity of the fluid

(iv) The temperature profile decreases with increasing values of both Prandtl number and Eckert number

(v) Magnitude of skin friction decreases with an increase in unsteadiness parameter and increases with an increase in magnetic parameter

(vi) Nusselt number decreases with an increase in Prandtl number and Eckert number

\section{Data Availability}

No data were used to support this study.

\section{Conflicts of Interest}

The authors declare that there are no conflicts of interest regarding the publication of this paper. 


\section{Acknowledgments}

The authors would like to sincerely appreciate funding from Researchers Supporting Project (no. RSP-2020/58), King Saud University, Riyadh, Saudi Arabia. R. Ali acknowledges the School of Art and Sciences, University of Central Asia, Kyrgyz Republic, for travel grant to visit the Department of Basic Sciences, UET, Taxila, and CFS, Dusseldorf.

\section{References}

[1] B. C. Sakiadis, "Boundary-layer behavior on continuous solid surfaces: II. The boundary layer on a continuous flat surface," AIChE Journal, vol. 7, no. 2, pp. 221-225, 1961.

[2] L. J. Crane, "Flow past a stretching plate," Zeitschrift für angewandte Mathematik und Physik ZAMP, vol. 21, no. 4, pp. 645-647, 1970.

[3] P. S. Gupta and A. S. Gupta, "Heat and mass transfer on a stretching sheet with suction or blowing," The Canadian Journal of Chemical Engineering, vol. 55, no. 6, pp. 744-746, 1977.

[4] P. Carragher and L. J. Crane, "Heat transfer on a continuous stretching sheet," ZAMM-Zeitschrift für Angewandte Mathematik und Mechanik, vol. 62, no. 10, pp. 564-565, 1982.

[5] A. Shahzad, R. Ali, M. Hussain, and M. Kamran, "Unsteady axisymmetric flow and heat transfer over time-dependent radially stretching sheet," Alexandria Engineering Journal, vol. 56, no. 1, pp. 35-41, 2017.

[6] A. Shahzad and R. Ali, "MHD flow of a non-Newtonian Power law fluid over a vertical stretching sheet with the convective boundary condition," Walailak Journal of Science and Technology (WJST), vol. 10, no. 1, pp. 43-56, 2012.

[7] A. Shahzad and R. Ali, "Approximate analytic solution for magneto-hydrodynamic flow of a non-Newtonian fluid over a vertical stretching sheet," Canadian Journal of Applied Sciences, vol. 2, pp. 202-215, 2012.

[8] H. I. Anderson, J. B. Aarseth, and B. S. Dandapat, "Heat transfer in a liquid film on an unsteady stretching surface," International Journal of Heat and Mass Transfer, vol. 43, pp. 69-74, 2000.

[9] C. Wang, "Analytic solutions for a liquid film on an unsteady stretching surface," Heat and Mass Transfer, vol. 42, no. 8, pp. 759-766, 2006

[10] M. S. Abel, N. Mahesha, and J. Tawade, "Heat transfer in a liquid film over an unsteady stretching surface with viscous dissipation in presence of external magnetic field," Applied Mathematical Modelling, vol. 33, no. 8, pp. 3430-3441, 2009.

[11] R. C. Aziz and I. Hashim, "Liquid film on unsteady stretching sheet with general surface temperature and viscous dissipation," Chinese Physics Letters, vol. 27, no. 11, Article ID 110202, 2010.

[12] R. C. Aziz, I. Hashim, and A. K. Alomari, “Thin film flow and heat transfer on an unsteady stretching sheet with internal heating," Meccanica, vol. 46, no. 2, pp. 349-357, 2011.

[13] M. M. Nandeppanavar, K. Vajravelu, M. Subhas Abel, S. Ravi, and H. Jyoti, "Heat transfer in a liquid film over an unsteady stretching sheet," International Journal of Heat and Mass Transfer, vol. 55, no. 4, pp. 1316-1324, 2012.

[14] M. Z. Salleh, R. Nazar, and I. Pop, "Boundary layer flow and heat transfer over a stretching sheet with Newtonian heating," Journal of the Taiwan Institute of Chemical Engineers, vol. 41, no. 6, pp. 651-655, 2010.
[15] Hartmann, "Flow of electrically conducting fluid over a flat plate in the presence of a transverse magnetic field," NACA Technical Report 3071, NACA, Abuja, Nigeria, 1957.

[16] A. Ishak, R. Nazar, and I. Pop, "MHD boundary-layer flow due to a moving extensible surface," Journal of Engineering Mathematics, vol. 62, no. 1, pp. 23-33, 2008.

[17] M. S. Abel, J. Tawade, and M. M. Nandeppanavar, "Effect of non-uniform heat source on MHD heat transfer in a liquid film over an unsteady stretching sheet," International Journal of Non-linear Mechanics, vol. 44, no. 9, pp. 990-998, 2009.

[18] Z. Abbas, T. Javed, M. Sajid, and N. Ali, "Unsteady MHD flow and heat transfer on a stretching sheet in a rotating fluid," Journal of the Taiwan Institute of Chemical Engineers, vol. 41, no. 6, pp. 644-650, 2010.

[19] T. Hayat, M. Qasim, and S. Mesloub, "MHD flow and heat transfer over permeable stretching sheet with slip conditions," International Journal for Numerical Methods in Fluids, vol. 66, no. 8, pp. 963-975, 2011.

[20] C.-H. Chen, "On the analytic solution of MHD flow and heat transfer for two types of viscoelastic fluid over a stretching sheet with energy dissipation, internal heat source and thermal radiation," International Journal of Heat and Mass Transfer, vol. 53, no. 19-20, pp. 4264-4273, 2010.

[21] M. A. Fadzilah, R. Nazar, M. A. Norihan, and I. Pop, “MHD boundary layer flow and heat transfer over a stretching sheet with induced magnetic field," Heat and Mass Transfer, vol. 47, pp. 155-162, 2011.

[22] F. Labropulu, "Unsteady stagnation-point flow of a Newtonian fluid in the presence of a magnetic field," International Journal of Non-linear Mechanics, vol. 46, no. 7, pp. 938-941, 2011.

[23] W. Ibrahim and B. Shanker, "Unsteady MHD boundary-layer flow and heat transfer due to stretching sheet in the presence of heat source or sink," Computers \& Fluids, vol. 70, pp. 21-28, 2012.

[24] D. Bhukta, G. C. Dash, S. R. Mishra, and S. Boag, "Dissipation effects on MHD mixed convection flow over a stretching sheet through porous medium with non-uniform heat source sink," Ain Shams Engineering Journal, vol. 8, no. 3, pp. 353-361, 2017.

[25] M. Gnaneswara Reddy, P. Padma, and B. Shankar, "Effects of viscous dissipation and heat source on unsteady MHD flow over a stretching sheet," Ain Shams Engineering Journal, vol. 6, no. 4, pp. 1195-1201, 2015.

[26] K. Anantha Kumar, V. Sugunamma, N. Sandeep et al., "Simultaneous solutions for first order and second order slips on micropolar fluid flow across a convective surface in the presence of Lorentz force and variable heat source/sink," Scientific Reports, vol. 9, p. 14706, 2019.

[27] K. Anantha Kumar, V. Sugunamma, and N. Sandeep, "Effect of thermal radiation on MHD Casson fluid flow over an exponentially stretching curved sheet," Journal of Thermal Analysis and Calorimetry, vol. 140, no. 5, pp. 2377-2385, 2020.

[28] K. Anantha Kumar, N. Sandeep, V. Sugunamma, and I. L. Animasaun, "Effect of irregular heat source/sink on the radiative thin film flow of MHD hybrid ferrofluid," Journal of Thermal Analysis and Calorimetry, vol. 139, no. 3, pp. 21452153, 2020.

[29] K. Anantha Kumar, V. Sugunamma, and N. Sandeep, "Influence of viscous dissipation on MHD flow of micropolar fluid over a slendering stretching surface with modified heat flux model," Journal of Thermal Analysis and Calorimetry, vol. 139, no. 6, pp. 3661-3674, 2020. 
[30] K. Anantha Kumar, V. Sugunamma, and N. Sandeep, "Physical aspects on unsteady MHD free convective stagnation point flow of micropolar fluid over a-stretching surface," Heat Transfer-Asian Research, vol. 48, no. 8, pp. 3968-3985, 2019.

[31] K. Anantha Kumar, V. Sugunamma, and N. Sandeep, "A nonFourier heat flux model for magnetohydrodynamic micropolar liquid flow across a coagulated sheet," Heat TransferAsian Research, vol. 48, no. 7, pp. 2819-2843, 2019.

[32] K. Anantha Kumar, V. Sugunamma, N. Sandeep, and J. V. Ramana Reddy, "Numerical examination of MHD nonlinear radiative slip motion of non-Newtonian fluid across a stretching sheet in the presence of porous medium," Heat Transfer Research, vol. 50, no. 12, pp. 1163-1181, 2019.

[33] K. Anantha Kumar, J. V. Ramana Reddy, V. Sugunamma, and N. Sandeep, "Magnetohydrodynamic Cattaneo-Christov flow past a cone and a wedge with variable heat source/sink," Alexandria Engineering Journal, vol. 57, no. 1, pp. 435-443, 2018.

[34] K. Anantha Kumar, V. Sugunamma, and N. Sandeep, "Impact of non-linear radiation on MHD non-aligned stagnation point flow of micropolar fluid over a convective surface," Journal of Non-equilibrium Thermodynamics, vol. 43, no. 4, pp. 327-345, 2018. 